\title{
Derived scales for degree of simultaneous contrast in six Benussi ring figures
}

\author{
NANCY S. ANDERSON, STEVEN M. PINE, and AZRIEL ROSENFELD \\ University of Maryland, College Park, Maryland 20742
}

\begin{abstract}
Gestalt psychologists have explained the reduction of simultaneous contrast in the Benussi ring figure as a result of laws of mental organization, i.e., "good continuation." The present study investigated the properties of these laws by studying six variations of the basic Benussi figure, which varied with respect to the type of boundary used to divide the ring. Scale values related to the dimension of degree of simultaneous contrast were derived using the method of paired comparisons. The results supported an explanation based on "apparent" separation between "parts" of the figure and degree of figure-ground symmetry rather than "good continuation."
\end{abstract}

When a gray stimulus ring is placed on a half-black, half-white background, the part viewed against the white background appears darker than the part viewed against a black background. When the gray ring is bisected by a dark line at the boundary between the black and white regions, the effect is enhanced. This simultaneous contrast effect can be explained in terms of neural inhibition at the retinal level (Ratliff, Hartline, \& Miller, 1963), and it has been shown that the differences in excitation are a function of size, luminance of background, and test fields; and the separation between the fields (Diamond, 1960, 1962; Heinemann, 1955).

When the gray stimulus ring is not bisected, it tends to appear uniformly gray despite the effects of simultaneous contrast. This phenomenon was studied in some depth by the Gestalt psychologist, Koffka (1935), who named this stimulus arrangement the Benussi ring, after the original investigator of the effect (it is also referred to as the Koffka ring). Koffka suggested that the phenomenon could be explained as a higher order mental effect in terms of laws of mental organization. According to Koffka's first law of unit formation and segregation $(1935$, p. 126), proximal stimulation which consists of several different areas of homogeneous stimulation organizes itself such that areas which receive the same stimulation form unitary field parts segregated from the other areas of stimulation. Equality of stimulation produces "forces of cohesion," and inequality of stimulation produces "forces of segregation." For the unbisected Benussi ring, the "forces of cohesion" which hold the ring together are so strong as to overcome the segregating forces of the black and white backgrounds, i.e., simultaneous contrast.

This research was supported in part by a grant from the National Science Foundation, GJ-32258X, and in part by a grant from the Biomedical Sciences Support Center of the University of Maryland to the Center for Language and Cognition. Requests for reprints should be sent to Nancy S. Anderson, Department of Psychology, University of Maryland, College Park, Maryland 20742.
Recent investigations into the Benussi ring effect have supported the Gestalt claim regarding the role of organization and postretinal factors. Julesz (1971) used a computer to generate stimuli which, when viewed as a stereo-pair, produced Benussi ring figures. Subjects reported depth effects in the figure that were visible only with binocular viewing, thus implying postretinal effects. Wist \& Susen (1973) studied the depth effect by moving the bisecting line out of the plane of the figure. They, also, found a difference between binocular and monocular viewing. Other investigations (Berman \& Leibowitz, 1965; Cohen, Bill, \& Gilinsky, 1968; Mackavey, 1969) have studied different aspects of the figure's organization and have, likewise, concluded that postretinal effects must be involved.

The present study attempted to explicate these higher order effects by following the Gestalt line of thought to search for the rules of organization which govern the perceived darkness of the gray ring in the Benussi figure. In particular, the effects of varying the type of boundary used to cut the gray stimulus ring were studied on subjects' judgments of the apparent contrast.

\section{METHOD}

\section{Stimuli}

The six Benussi ring figures shown in Figure 1 were used as stimuli. The stimuli differ only with respect to the type of boundary used to separate the black and white regions.

The black, white, and gray parts of the six figures were cut from the same three sheets of Colortone construction paper to assure uniform luminance. When viewed by the subjects at the average distance of $129 \mathrm{~cm}$, the black regions subtended a visual angle of $10 \mathrm{deg} 42 \mathrm{~min} \times 10 \mathrm{deg}$ of arc $(9.5 \times 8.9 \mathrm{~cm})$; the white, $8 \mathrm{deg} 34 \mathrm{~min} \times 4 \mathrm{deg} 18 \mathrm{~min}$ of arc $(7.6 \times 3.8 \mathrm{~cm})$. The gray annuli were squares subtending visual angles of $5 \mathrm{deg}$ of arc $(4.5 \mathrm{~cm})$ along each edge with an annulus width of $54 \mathrm{deg}$ arc $(7.9 \mathrm{~mm})$. The average luminances of the figures were $6.3,40.0$, and 22.5 footcandles for the black, white, and gray regions, respectively.

\section{Design and Procedure}

The method of paired comparisons was used to scale the stimuli with respect to the degree of illusionary effect produced 


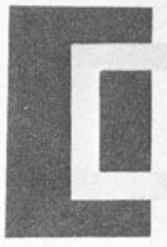

(a)

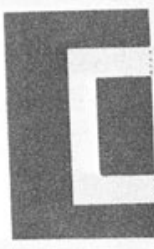

(c)

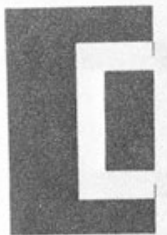

(e)

Figure 1. Six Benussi ring figures.

by the various boundary types. The stimuli were laid out in a row, two at a time, on a well-lit table (40 footcandles) at which the subject was seated. Each subject made comparisons for all possible combinations of the six pictures taken two at a time for each condition of the study, i.e., 15 comparisons per condition. The study consisted of two experimental and one control condition, each of which is explained below.

"Bar" condition. Subjects based their comparisons on the vertical portion of the gray rectangular annulus centered in the white background region of each stimulus. On each trial, the subject responded either "left" or "right," depending on which of the pair of two stimuli appeared to have the darker vertical portion of the gray rectangular annulus designated as the "bar."

"Difference" condition. Subjects were instructed to compare the gray "bars" in the white and black regions of each stimulus and to indicate for which stimulus there appeared to be the greatest difference in darkness between "bars."

Control condition. A black jacket cover, cut from the same sheet of Colortone paper as the black areas of the stimuli, was slipped over each stimulus. When the covers were in place, only the half of the gray annulus in the white region could be seen. In addition, the boundary types which divided the gray rings were also covered by the jacket covers. The subject responded left or right in the same way as used in the "Bar" condition. Each subject was run in two paired comparison conditions; half of the subjects with the control and "Bar" conditions and half with the control and "Difference" conditions. Stimulus presentation order was random, and the order in which the control and "Bar" or "Difference" conditions occurred was counterbalanced over subjects.

\section{Subjects}

Eight-eight University of Maryland undergraduates served as subjects. None had prior experience with the task, and each participated in return for extra credit points in an undergraduate psy chology course.

\section{RESULTS}

The number of intransitivities which occurred in the preference data from each subject was used as a measure of response consistency. An intransitivity occurred when, for the three stimuli $i, j, k$ : i was judged darker than $\mathrm{j}$, and $\mathrm{j}$ darker than $\mathrm{k}$, but $\mathrm{i}$ was not judged darker than $\mathrm{k}$. On the average, there were more than twice as many intransitivities made in the "Bar" condition than in the "Difference" condition, 3.25 compared to 1.5 . The average number of intransitivities made in the control condition was 4.12 . The expected number of intransitivities for randomly made judgments is five (Kendall, 1955; p. 157).

Preference data for the six stimuli were summed over 44 subjects for each condition. In the control condition, the boundary variations between the stimuli were hidden; therefore, all stimuli in this condition should have been preferred over the other stimuli an equal number of times. A chi square test of independence was performed on the six sums representing the number of times one stimulus was preferred over all others. Chi square $\left(\chi^{2}=p>.05\right)$ was not significant for the control condition; however, the chi squares performed on the experimental conditions were significant, $\chi^{2}(5)=19.3$, $\mathrm{p}<.005$; and $\chi^{2}(5)=137.2, \mathrm{p}<.001$ for the "Bar" and "Difference" conditions, respectively. The results of these statistical tests indicated that no systematic bias existed between the six stimuli.

Thurstone's scale for paired comparisons, Case V, was applied to scale the two comparison conditions, and the derived scales are shown in Figure 2. As a further check, the Guttman-Lingoes nonmetric scaling program (SSA II) was used on the same data. In one dimension, the scales generated from the program were virtually identical to the scales obtained by Thurstone's method. (In higher dimensional spaces, perfect fits were obtained in four dimensions for the "Bar" condition and in two dimensions for the "Difference" condition.)

\section{DISCUSSION}

In the control condition, all six stimuli should have appeared to be the same since the only difference between the figures was in the boundary type that divided the gray ring, and this was hidden by the jacket that was used to cover half of each figure. The results from the control condition confirmed that there were no apparent brightness differences between the gray rings for the six stimuli when the boundaries were covered. Therefore, the method of this study was successful in eliminating any bias between the stimuli, and the derived scales can be taken as a measure of the degree of simultaneous contrast as a function of the six boundary types.

As might be expected, the derived scales for the "Difference" and "Bar" conditions were similar. There were, however, basic differences between the scales which need to be explained. First, the relative distances between the stimuli were much smaller in the "Bar" scale than in the "Difference" scale. Secondly, the order of two pairs of stimuli, those at each of the extreme ends of the two scales, were reversed. Both the smaller interstimulus spread of the "Bar" scale and the stimulus reversals between scales can probably be explained by the fact that there were 
twice as many intransitivities committed in the "Bar" condition. Apparently, the subjects had a more difficult time in making a preference judgment for this case. Another indication of this difficulty is found in the number of dimensions needed to render a perfect fit to the data when the nonmetric scaling program was used; twice as many dimensions were needed in the "Bar" condition, i.e., four vs. two.

A logical explanation for the differences between the conditions can be based on the physical dimensions of the stimuli used. The visual angle subtended by the entire Benussi figure was about $6 \mathrm{deg}$ of arc. This is considerably more than the human eye is capable of focusing on in one fixation. However, the "Bar" alone subtended a visual angle of only 1 deg of arc, well within what the eye can attend to in a single fixation. In the "Bar" condition, subjects were required only to study the "bar" in the white region for each stimulus; whereas, in the "Difference" condition, subjects were required to attend to both the "bar" in the white and the "bar" in the black area for both stimuli. Therefore, it is reasonable to assume that, in the "Bar" condition, the black and white inducing regions may not have been as consistently attended to as in the "Difference" condition.

The rank order of the "Difference" scale is almost identical to the order obtained during a pilot study using photographs of computer-generated pictures. (The only difference between the two results was in the order of the Midline and Notch stimuli, and since the results of the present study were based on 88 subjects rather than 10 subjects in the pilot study, the ordering of stimuli by the present data is preferred.) Because of the consistency between these two studies and for the reasons discussed above, the remainder of this paper will concern itself with the scale derived from the "Difference" condition.

A common Gestalt explanation of how the degree of simultaneous contrast changes as a function of variations in the Benussi figure has been "good continuation." According to the first law of unit formation and segregation stated in this paper, "good continuation" generates "forces of cohesion" within the figure which are capable of overcoming the forces of simultaneous contrast. One way of interpreting "good continuation" here is in terms of "connectedness." Several researchers (Berman \& Leibowitz, 1965; Julesz, 1971) have hypothesized that the more contact that exists between the halves of the ring, the smaller $w$ ill be the degree of contrast between them. In the present study, the results from two of the six stimuli can be explained in terms of connectedness. The figure with the smallest apparent darkness difference between halves of the gray annulus ring, "No Line," had the greatest degree of connectedness between the halves, that is, one continuous gray ring. Also consistent with the hypothesis of connectedness was the fact that the figure with the worst "connectedness" (least continuation), "Midline," was near the opposite end of the scale from "No Line." But, this is as far as connectedness can be used to explain the judgments. The derived scale values of two of the stimuli, "Line in White" and "Notch," were such as to eliminate the notion of "connectedness" as a viable explanation.

Notch had the highest scale value on the "Difference" scale. It was judged to have a considerably greater degree of contrast than even Midline. This result cannot be explained only by the hypothesis of "connectedness," since Notch has a higher degree of "connectedness" than Midline. In fact, Midline is usually described in the literature as producing the maximum simultaneous contrast (Berman \& Leibowitz, 1965; Gregory, 1969; Koffka, 1935; Wist \& Susen, 1973). However, with the proper interpretation of the notches in "Notch," this paradox can be resolved and the derived scale supported by previous research. Both Berman and Leibowitz (1965) and Mackavey (1969) have shown that the contrast between two fields (in this case, the halves of the ring) is enhanced as a function of the separation between them. In the Berman and Leibowitz study, the

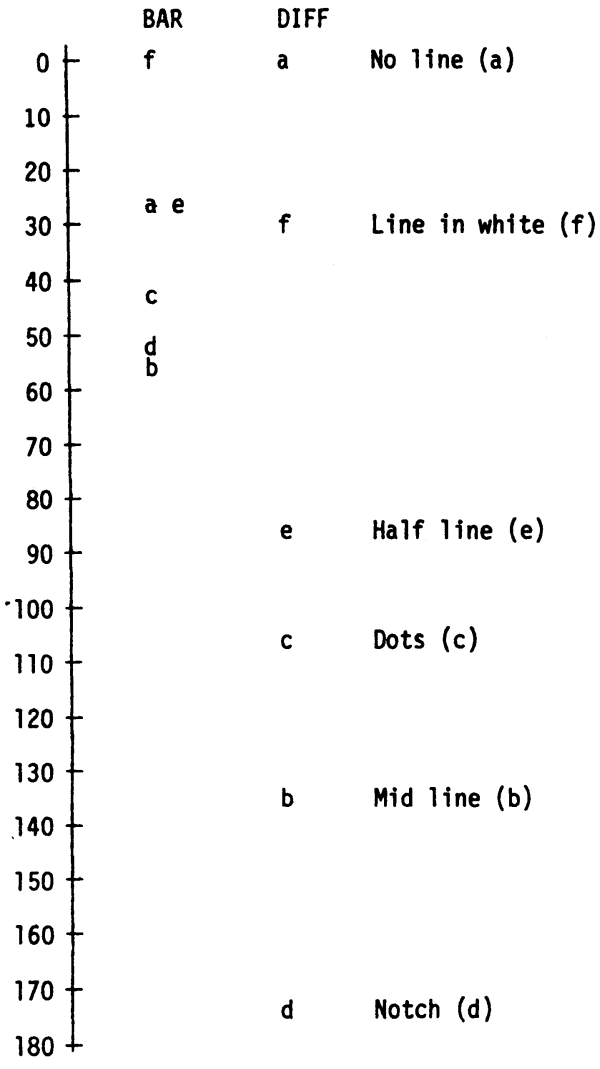

Figure 2. Thurstone scale values for the six Benussi ring stimuli for both "Bar" and "Difference" methods.

thickness of the bisecting line was varied, with the effect of greater contrast as the thickness of the line was increased. The notches in "Notch" could be considered analogous to a thickening of the top and bottom thirds of the line in "Midline." Therefore, the notches gave the appearance of a greater separation between the halves of the ring, as did the thickening of the line in the Berman and Leibowitz study. Apparently, the appearance of separation between parts of the ring reduces the "forces of cohesion."

With respect to the "Half line" and "Dots" stimuli, it is not clear that their positions can be explained in terms of the appearance of separation between the halves of the gray ring. Perhaps separation plays a lesser role for these types of boundaries. Yet, their rank order precludes any explanation in terms of connectedness, since "Dots" has the greater degree of "connectedness" but was ranked above "Half Line."

The results for the stimulus with the line moved into the white region is intriguing because it was low on the scale despite the fact that its line effectively divided the ring into two parts. Again, "connectedness" cannot account for this fact, since "Line-in-white" and "Midline" had the same degree of "connectedness." The only difference between these stimuli was a shift in the position of the cut. Therefore, the differences in scale values between these two must be due to the position of the cut. Since the position of the cut is relative to the black and white regions and these correspond to "ground" in the Gestalt sense, this can be taken as evidence that figure-ground in some way affects simultaneous contrast.

Although it is true that each of the stimulus arrangements divide themselves nicely into figure and ground, this alone cannot account for the results. Five of the six figures are 
essentially identical with respect to the shape and orientation of figure-ground. In these stimuli, therefore, figure-ground could not have played an important role in producing the observed scale. Only the stimulus with the cut shifted into the white region has a substantially different look with respect to figure-ground. We suggest that the degree of simultaneous contrast was weakened in this stimulus by the asymmetry caused by the failure of the cut in the ring (figure) to align with the cut between the black and white regions (ground).

\section{SUMMARY}

Although retinal inhibition is important in producing simultaneous contrast at the retinal level, this study supports the previous research which demonstrates the importance of higher order mental effects. In the Benussi ring figures, the line which was used to create the various boundary types was so thin that it could not possibly have a retinal effect. Yet, results have shown that these minute variations were sufficient to produce a scale of apparent differences in the degree of simultaneous contrast and that the derived scale will vary depending on how subjects are instructed to view the stimuli.

In addition to supporting previous research, we have sneaked a look at the laws by which these higher order effects operate. It is clear that laws of mental organization are important in establishing the degree to which the simultaneous contrast is cancelled. In particular, we have seen that the Gestalt concept of "good continuation," interpreted as "connectedness," is not capable of explaining the derived scales. It would seem, however, that the appearance of separation between parts of the figure can act to enhance simultaneous contrast; whereas, asymmetry between the figure and ground can reduce the contrast.

\section{REFERENCES}

Berman, P. W., \& Leibowitz, H. W. Some effects of contour on simultaneous brightness contrast. Journal of Experimental Psychology, 1965, 69, 251-256.

Cohen, H. H., Bill, J. C., \& Gilinsky, A. S. Simultaneous brightness contrast: Variations of Koffka's ring. Proceedings of the 76th Annual Convention of the American Psychological Association, 1968, 99-100.

Diamond, A. L. A theory of depression and enhancement in the brightness response. Psychological Review, 1960, 67, 168-198.

Diamond, A. L. Simultaneous contrast as a function of testfield area. Journal of Experimental Psychology, 1962, 64, 336-345.

GrEgory, R. L. Eye and brain. New York: McGraw-Hill, 1966.

Heinemann, E. G. Simultaneous brightness induction as a function of inducing and test-field luminances. Journal of Experimental Psychology, 1955, 50, 89-96.

Julesz, B. Foundations of cyclopean perception. Chicago: University of Chicago Press, 1971.

Kendall, M. G. Rank Correlation Methods (2nd ed.). New York: Hafner, 1955.

KoFfKA, K. Principles of gestalt psychology. New York: Harcourt, Brace, 1935.

MACKAVEY, W. R. Spatial brightness changes in Koffka's ring. Journal of Experimental Psychology, 1969, 82, 405-409.

Ratliff, F., Hartline, H. K., \& Miller, W. H. Spatial and temporal aspects of retinal inhibitory interaction. Journal of the Optical Society of America, 1963, 53, 110-120.

WIST, E. R. \& Susen, P. Evidence for the role of post-retinal process in simultaneous contrast. Psychologische Forschung, 1973, 36, 1-12.

(Received for publication May 30, 1975.) 